\title{
Retention in Methadone Maintenance Treatment: A Preliminary Analysis of the Role of Transfers Between Methadone Prescribing Physicians
}

\author{
Carol J. Strike ${ }^{*}, 1,2$, William Gnam ${ }^{1,2}$, Karen Urbanoski ${ }^{1}$, Benedikt Fischer ${ }^{1,3}$, David Marsh ${ }^{4}$ and \\ Margaret Millson ${ }^{2,5}$
}

${ }^{I}$ Centre for Addiction and Mental Health, Toronto, Canada

${ }^{2}$ University of Toronto, Toronto, Canada

${ }^{3}$ Centre for Addictions Research of British Columbia, Victoria, Canada

${ }^{4}$ Vancouver Coastal Health Authority, Vancouver, Canada

${ }^{5}$ Ontario HIV Treatment Network, Toronto, Canada

\begin{abstract}
We examine the impact of transfers between methadone treatment prescribing physicians on 1-year retention. Episode data ( $\mathrm{n}=13,359 ; 1996$ to 2001) drawn from a Canadian population-based methadone registry were analysed using a binary logistic regression model. The odds ratios for remaining in treatment for 365 days or more decreased for repeated episodes of treatment, but increased with age and number of transfers. The positive effect of the number of transfers was non-linear and tended to level off with higher numbers of transfers. Analyses indicate that transfers between prescribing physicians may play an important role in prolonging methadone maintenance treatment.
\end{abstract}

Keywords: Methadone, treatment retention, treatment outcome, treatment systems, population-based.

\section{INTRODUCTION}

Evidence consistently shows that patients who remain longer in methadone maintenance treatment (MMT) for opioid dependence are more likely to reduce illicit drug use and criminal activity [1-7]. For example, the Drug and Alcohol Treatment Outcome Studies (DATOS) in the United States showed that patients reported significantly less heroin use and fewer drug injections following 12 months or more of treatment as opposed to those who stayed less than 12 months [8]. However, many patients drop-out or leave before completing 12 months of treatment and many return for treatment at a later date $[9,10]$.

Given that MMT outcomes improve with longer duration, it is important to develop MMT systems that encourage patients to remain for longer periods of time, and perhaps discourage cycling in and out of treatment. Previous studies have shown that many factors influence retention in MMT, including: adequate dose, age, treatment episode number, provider type, patient satisfaction with treatment, referrals for medical and mental health services and the number, training and experience of staff [5-8, 10-12]. At a systems level, facilitating transfers between MMT prescribing physicians may improve treatment retention. Patients may benefit from transfers if instead of facing a discharge and search for other treatment slots they can transfer to another methadone prescribing physicians. Reasons for transfer may include : a desire for a different or more therapeutic relationship, physical relocation and incarceration/release into the community.

*Address correspondence to this author at the Centre for Addiction and Mental Health, Toronto, Canada; Tel: 416535 8501; Fax: 416979 4703;

E-mail: Carol_Strike@camh.net
Previous studies have not examined the impact on retention of transfers between MMT prescribing physicians during a continuous treatment episode. Understanding the impact of transfers on treatment outcome, including duration, is important from the perspective of drug treatment system design. If the impact is positive, treatment systems could improve patient outcomes by allowing and facilitating transfers.

Using data from the Ontario population-based patient registry, we answer the following question: do patients who transfer between MMT prescribing physicians remain in treatment longer than other patients? In this system, transfers between MMT prescribing physicians can occur in three circumstances: physical relocation of patients that requires a transfer of care; incarceration/release from custody that requires a transfer of care to a corrections physician or a community physician on release; patient- or provider-initiated transfer when they do not share treatment goals (e.g., transfer from a higher threshold to a lower threshold). There are no MMT stabilization clinics in this system so patients do not transfer from stabilization clinics to other MMT practices. A government-funded, universal health insurance program reimburses physicians for MMT and for most patients methadone is paid for by social assistance programs (i.e., government funded welfare or disability benefits) or by private drug insurance companies (i.e., employment-related benefit programs). Only a small minority pay for methadone out-ofpocket. In Ontario, patients can initiate and complete a transfer between MMT or any other medical care provider where available.

\section{METHODS}

MMT patient registration data from the College of Physicians and Surgeons of Ontario (CPSO) Methadone Mainte- 
nance Registry of Patients and the Registry of Methadone Prescribing Physicians for MMT between January 1, 1996 and December 31, 2001 were used for the analysis. As stipulated by legislation, prescribing physicians in Ontario are required to submit a registration form each time a patient begins and ends MMT for opioid dependence. Physicians who prescribe methadone for pain management do not submit registrations [13]. The registry collects a limited amount of information about methadone patients and their treatment but provides a unique population-based longitudinal data source recording the duration of treatment episodes, as well as characteristics of the prescribing physicians.

A total of 13,823 MMT episodes were available in the CPSO dataset. We deleted 12 episodes in correctional facilities that were not preceded or succeeded by treatment in the community because the length of sentence and not the natural history of voluntary MMT determined the duration of treatment. After excluding 244 episodes whose termination dates were identical to the start dates, and 208 episodes with missing data, 13,359 episodes of MMT were available for analysis.

The unit of analysis is the treatment episode. The total number of treatment episodes exceeds the number of individuals in-treatment because of repeat registrations in a given year (i.e., patients may initiate and discontinue treatment multiple times). During a continuous episode of MMT when a patient transfers from one physician or facility (e.g., correctional facility) to another, the transfer is recorded in the CPSO Registry as a cessation and a new registration. We used registration and discharge dates and physician unique identifiers for treatment episodes to create a transfer variable. In some cases, the discharge/re-registration process may create an administrative registration gap but not a treatment gap. For example, there may be a short delay between registration of the discharge and re-registration of the patients by the new MMT physician. We followed the approach used by Bell et al. 2006 [9] to identify discharges versus transfers. In terms of MMT prescribing patterns in Ontario, registration gaps of 7 days or less likely represent only an administrative gap (i.e., discharge and re-registration) but without a gap in a methadone prescription. Registration gaps of 7 days or more are most likely accompanied by a gap in methadone prescriptions meaning that patients are without methadone during this period of time. A gap of 7 days or less between a discharge and a new registration for the same patient was coded as a transfer - i.e., a continuous single episode of treatment with multiple prescribing physicians. A gap in registration of greater than 7 days was coded as a discharge and the termination of an episode of continuous MMT. Unfortunately, the registry data does not capture reason for a transfer or include a variable indicating a transfer.

Patients could have several MMT prescribing physicians of different types (i.e., correctional facility, independent physician or group practice) during the course of a continuous treatment episode. Six provider-type variables were created to reflect the combinations of provider types observed within the data. A change in federal correctional policy in 1998 and Ontario provincial correctional policy in 1999 required that those on MMT at the time of incarceration be maintained on MMT throughout their incarceration unless there was a medical reason justifying discontinuation of treatment. Thus there are significant increases in treatment episodes in correctional facilities after these policy shifts.

Covariate selection was based upon a literature review of the relevant individual and program characteristics associated with treatment retention, and the variables available in the dataset (date of registration, date of cessation, date of death, age, gender, region and provider and provider type). Data on dose and reason for cessation were not available. To model the determinants of staying in treatment for 365 days or longer, we estimated a binary logistic regression model, using an indicator of treatment duration of 365 days or greater as the dependent variable. Binary logistic regression is used when the dependent variable has two values (e.g., 0 or 1; yes versus no). We included as predictor variables: age (single years); age squared; sex (male); number of transfers; number of transfers squared; region (coded as 6 dummy variables, with metropolitan Toronto as the reference category); treatment episode provider type (coded as dichotomous variables: group practice-only, independent physicianonly, group practice and correctional facility, group practice and independent physician, independent physician and correctional facility, and independent physician and correctional facility and group practice); and a count variable for patient episode number within the dataset (numbered chronologically from 1 onwards). A linear and quadratic time trend measured in years was included to control for possible secular changes in MMT practice over time. Because the possibility of transferring between prescribing physicians varied across the province, pre-specified interaction terms were included and tested for significance using a global likelihood ratio test; all interaction terms were retained in the final model. Only variables shown to be significant variables are presented in Table 1. Huber-White robust standard errors were estimated to account for clustering within the data (i.e, repeated observations on the same patient). All statistical analyses were conducted using STATA SE version 8 software. The Ethics Board at the Centre for Addiction and Mental Health approved the study.

\section{RESULTS}

The majority of treatment episodes $(54.3 \%)$ were for males, and the mean age at treatment registration was 34.9 years, with a range of 14 to 74 years. Of the total number of treatment episodes, $56.4 \%$ exceeded 364 days. The number of transfers ranged from: 0 to 5, with a mode of 0 .

The odds ratio for transfer indicate that remaining in treatment for 365 days or more $(\mathrm{OR}=1.72$ - see Table $\mathbf{1})$ is greatly improved for patients with at least one transfer during their treatment episode. However, the odds ratio for the transfer variable indicates that the positive influence of transfers tends to level off with increasing number of transfers. The odds ratio for age indicates that remaining in treatment for 365 days or more also increased with age and year of registration. The positive and significant odds ratio associated with year of registration indicates that over the study interval there has been a significant increase in retention rates. Nevertheless, the odds ratio for repeat episodes of treatment indicates that remaining in treatment for 365 days or more decreased for repeat episodes.

The sensitivity analysis comparing transfer gaps of 5 days versus 14 days showed that the results were qualita- 
tively unchanged from those using 7 days to define a transfer versus a treatment break.

Table 1. Adjusted Odds Ratios ${ }^{\mathrm{a}}$ of Remaining in MMT for 365 Days or More by Selected Predictors

\begin{tabular}{|c|c|c|}
\hline Predictors & AOR & $95 \% \mathrm{CI}$ \\
\hline Age & 1.06 & 1.04 to 1.09 \\
\hline $\mathrm{Age}^{2}$ & 0.99 & 0.99 to 1.00 \\
\hline Sex $=$ male & 1.08 & 0.99 to 1.17 \\
\hline Episode $^{\mathrm{b}}$ & 0.84 & 0.79 to 0.89 \\
\hline Year of registration & 2.12 & 1.89 to 2.39 \\
\hline Year of registration ${ }^{2}$ & 0.94 & 0.93 to 0.94 \\
\hline Group practice & 1.06 & 0.18 to 6.34 \\
\hline Group practice and correctional & 0.88 & 0.15 to 5.10 \\
\hline Group practice and individual practitioner & 1.36 & 0.21 to 8.69 \\
\hline Individual practitioner and correctional & 0.53 & 0.10 to 2.93 \\
\hline Individual practice & 0.54 & 0.09 to 3.25 \\
\hline Number of transfers & 1.72 & 1.51 to 1.96 \\
\hline Number of transfers ${ }^{2}$ & 0.95 & 0.93 to 0.97 \\
\hline
\end{tabular}

episodes of treatment used for these analyses.

${ }^{\mathrm{b}}$ Maximum number of episodes $=7$.

\section{DISCUSSION}

Our population-based analyses show that more than half of patients are retained within the Ontario MMT system for 365 days or more with no treatment interruption of 7 or more consecutive days. However, these analyses also show that the probability varies by number of transfers, age and number of treatment episodes. As well, the odds ratios show that the probability of staying in treatment tends to level off with older age and with increasing number of transfers.

The manner in which transfers between MMT prescribing physicians influences treatment duration is difficult to determine from these analyses. It could be that transfers reflect movement of patients to MMT prescribing physicians with whom they can develop a productive therapeutic relationship. As well, transfers may indicate movement of patients between different thresholds of MMT practice. For example, an analysis of transfers from a Toronto-based MMT program found an increase in transfers for patients who were more likely to have drug-positive urines prior to transfer than those patients remaining in the program [14]. The finding that number of transfers is correlated with increased treatment retention suggests that ability to transfer between MMT prescribing physicians is a positive element of a treatment system and further research is needed to clarify the mechanism of this beneficial effect.

These analyses, combined with analyses of factors that influence retention to 730 days or more [10], suggest that age and number of treatment episodes are associated with both 1-year and 2-year MMT retention. Transfers appear to play a role for 1-year retention, whereas treatment setting type and region of treatment influence 2-year retention. These findings suggest the need for further research to determine if MMT systems can be designed to attend to the diverse needs of MMT patients over the course of their treatment.

For these analyses we used an administrative database that provided advantages in terms of cost, time, sample size and population representativeness. However, analyses were limited to the variables on the registration forms. While the sensitivity analyses showed that misclassification as a result of how transfers and breaks were approximated did not qualitatively change the results, a reliable variable in the source dataset would ensure that all transfers are captured and the rigour of our analyses improved. As well, a variable capturing data about the reason for transfer would improve our understanding of why and for whom transfers improve MMT retention.

Our results suggest that transfers are associated with improved retention. The extent to which these results are unique to the Ontario context or reflect trends among MMT patients in other settings is difficult to ascertain because of differences in health care and addiction treatment systems across jurisdictions, and also lack of comparable data. Comparison across jurisdictions, however, might provide insight into system-level factors that facilitate or impede MMT retention.

\section{ACKNOWLEDGEMENTS}

We thank the College of Physicians and Surgeons of Ontario for providing access to the registry databases and Denise Marigold for assistance with data management. The opinions expressed in this manuscript are those of the authors and not the College of Physicians and Surgeons of Ontario.

\section{REFERENCES}

[1] Cushman P Jr. Abstinence following detoxification and methadone maintenance treatment. Am J Med 1978; 65(1): 46-52.

[2] McGlothlin WH, Anglin MD. Shutting off methadone. Costs and benefits. Arch Gen Psychiat 1981; 38(8): 885-92.

[3] Gunne L, Gronbladh L. The Swedish methadone maintenance Program. In: Serban G, Ed. The Social and Medical Aspects of Drug-Abuse. New York, Spectrum Publications 1984; pp. 205-13.

[4] Rosenbaum MIJ, Murphy S. De facto destabilization as policy. The impact of short-term methadone maintenance. Contemp Drug Prob 1998; 15: 497-517.

[5] Ward J, Mattick R, Hall W. How long is long enough? Answers to questions about the duration of methadone maintenance treatment. In: Ward J, Mattick RP, Hall W, Eds. Methadone Maintenance Treatment and Other Opioid Replacement Therapies. Amsterdam, Harwood Academic Publishers 1998; pp. 305-36.

[6] Magura S, Nwakeze PC, Demsky SY. Pre- and in-treatment predictors of retention in methadone treatment using survival analysis. Addiction 1998; 93(1): 51-60.

[7] Zhang Z, Friedmann PD, Gerstein DR. Does retention matter? Treatment duration and improvement in drug use. Addiction 2003; 98(5): 673-84.

[8] Simpson DD, Joe GW, Brown BS. Treatment retention and followup outcomes in the Drug Abuse Treatment Outcome Study (DATOS). Psychol Addict Behav 1997; 11(4): 294-307.

[9] Bell J, Burrel T, Indig D, Gilmour D. Cycling in and out of treatment; participation in methadone treatment in NSW, 1990-2002. Drug Alcohol Depend 2006; 81: 55-61. 
[10] Strike C, Gnam W, Urbanoski K, Fischer B, Marsh D, Millson M. Factors predicting two-year retention in methadone maintenance treatment for opioid dependence. Addict Behav 2005; 30: 1025-8.

[11] Ball J, Ross A. The Effectiveness of Methadone Maintenance Treatment: Patients, Programs, Services and Outcomes. New York: Springer-Verlag 1991.
[12] Farré M, Mas A, Torrens M, Moreno V, Cami J. Retention rate and illicit opioid use during methadone maintenance interventions: a meta-analysis. Drug Alcohol Depend 2003; 65(3): 283-90.

[13] Brands J, Brands B, Marsh D. The Expansion of Methadone Prescribing in Ontario, 1996-1998. Addict Res 2000(a); 8: 485-96.

[14] Brands B, Blake J, Marsh DC. Changing patient characteristics with increased methadone maintenance availability. Drug Alcohol Depend 2000(b); 66(1): 11-20.

(C) Strike et al.; Licensee Bentham Open.

This is an open access article licensed under the terms of the Creative Commons Attribution Non-Commercial License (http://creativecommons.org/licenses/by$\mathrm{nc} / 3.0 /$ ) which permits unrestricted, non-commercial use, distribution and reproduction in any medium, provided the work is properly cited. 